\title{
Estrés social y rendimiento académico en adolescentes: proyecto DADOS
}

\author{
Alba Sales Chillida \\ al339353@uji.es \\ Mireia Adelantado Renau \\ adelantm@uji.es \\ Carlos Bou Sospedra \\ al379860@uji.es \\ María Reyes Beltrán Valls \\ vallsm@uji.es \\ Diego Moliner Urdiales \\ dmoliner@uji.es
}

LIFE Research Group, Universitat Jaume I, Castellón

\section{Resumen}

Introducción: La adolescencia constituye un periodo crítico del desarrollo caracterizado por múltiples cambios psicológicos, entre los que destaca un aumento del estrés social debido a las modificaciones en las relaciones interpersonales, que pueden influir en el rendimiento académico del adolescente. El objetivo del presente estudio fue analizar la relación entre el nivel de estrés social y el rendimiento académico en adolescentes.

Método: Un total de 269 alumnos de $2 .^{\circ}$ curso de educación secundaria obligatoria (ESO) (129 chicas y 140 chicos; 13,9 90,3 años), participantes del proyecto DADOS (Deporte, Adolescencia y Salud), fueron incluidos en el estudio. El estrés social se evaluó empleando el nivel 3 del cuestionario Behavior Assessment System for Children (BASC). Los estudiantes fueron dicotomizados en base a su nivel de estrés (normal $<60$ frente a elevado $\geq 60$ ). El rendimiento académico se evaluó mediante las calificaciones académicas finales de $1 .^{\circ}$ de ESO en matemáticas, castellano, valenciano, inglés y educación física y la media global.

Resultados: El nivel de estrés social estuvo inversa y significativamente asociado con todos los indicadores de rendimiento académico. Los análisis de covarianza indicaron que, excepto en la calificación de castellano, un estrés social elevado se relacionaba significativamente con un menor rendimiento académico. 
Conclusión: El nivel de estrés social parece estar inversamente asociado con el rendimiento académico en adolescentes. Los programas escolares enfocados a la mejora del rendimiento académico deberían considerar los posibles efectos negativos derivados de altos niveles de estrés social.

Palabras clave: salud, rendimiento académico, adolescencia, estrés social.

\section{Abstract}

Introduction: Adolescence is a crucial developmental period of life characterized by multiple psychological changes, such as increased stress levels due to modifications in interpersonal relationships, which could influence adolescents' academic performance. The aim of this study was to analyse the relationship between social stress levels and academic performance in adolescents.

Methods: A total of 269 students of the $2^{\text {nd }}$ academic year of secondary school (129 girls; $13.9 \pm 0.3$ years), participants from the DADOS (Deporte, Adolescencia y Salud) study, were included in the analyses. The level of social stress was evaluated through the Behavior Assessment System for Children (BASC) level 3 and dichotomized into normal or high ( $<60$ vs. $\geq 60$ ). Academic performance was assessed through the final school grades of the $1^{\text {st }}$ academic year of secondary school in Mathematics, Spanish, Valencian, English, Physical Education, and grade point average.

Results: The level of social stress was inversely associated with academic performance. Moreover, except for Spanish, analysis of covariance indicated that those adolescents with high levels of social stress showed lower academic performance, compared to their peers with normal levels of social stress.

Conclusion: The level of social stress seems to be inversely associated with academic performance in adolescents. School-based programs aimed to improve academic performance should consider the possible adverse effects derived from high levels of social stress.

Keywords: health, academic performance, adolescence, social stress.

\section{Introducción}

El estrés puede definirse como aquella reacción del ser humano ante situaciones amenazantes o de excesiva demanda (Vales 2009). Estudios previos han sugerido que el estrés podría jugar un papel fundamental en la etiología y la progresión de enfermedades cardiometabólicas como la obesidad, la hipertensión o la diabetes (Cruz et al. 2015), así como aumentar la probabilidad de padecer alteraciones del bienestar psicológico como la depresión y la ansiedad (Yeresyan y Lohaus 2014).

La adolescencia constituye un periodo crítico en el desarrollo caracterizado por múltiples cambios psicológicos (Ortega, Ruiz, Castillo y Sjöström 2008), entre los que destaca un aumento del estrés social, que es aquel debido a las modificaciones en las relaciones interpersonales (Van Roekel et al. 2015). Estudios recientes sugieren que la exposición a altos niveles de estrés también podría influir sobre la función cognitiva (Lupien, McEwen, Gunnar y Heim 2009), como por ejemplo sobre la memoria y los 
procesos de flexibilidad cognitiva, al alterar el funcionamiento del hipocampo y el córtex prefrontal del cerebro (Sandi 2013). No obstante, los estudios que han examinado la relación entre el estrés y el rendimiento académico en adolescentes han mostrado resultados contradictorios, ya que algunos han identificado una asociación negativa (Grasses y Rigo 2010; Fernández de Castro de León y Luévano Flores 2018), mientras que otros han reportado una falta de asociación (Gaeta González y Martín Hernández 2009).

Dado que la evidencia científica que relaciona el estrés con el rendimiento académico en adolescentes es limitada y contradictoria, centrándose principalmente en el estrés académico (Maturana y Vargas 2015), es fundamental ampliar el conocimiento sobre esta asociación. Por ello, el objetivo de nuestro estudio fue analizar la relación entre el nivel de estrés social y el rendimiento académico en estudiantes de $2 .^{\circ}$ curso de educación secundaria obligatoria (ESO).

\section{Método}

Participantes

Los participantes fueron seleccionados a través de clubs deportivos y de centros educativos de la provincia de Castellón (España). Los criterios de inclusión fueron los siguientes: estar matriculado en $2^{\circ}$ de ESO y no padecer ninguna enfermedad crónica. De los 274 adolescentes participantes en el estudio DADOS, 269 (129 chicas y 140 chicos; $13,9 \pm 0,3$ años) fueron incluidos en este estudio transversal.

\section{Procedimiento}

Este trabajo forma parte del proyecto de investigación DADOS (Deporte, Adolescencia y Salud), un estudio longitudinal de 3 años (2015-2018) cuyo objetivo principal es analizar la influencia de la práctica de actividad física sobre la salud física, el nivel de bienestar psicológico y el rendimiento académico durante la etapa de ESO. Los participantes acudían a las instalaciones de la Universitat Jaume I donde, bajo la supervisión de investigadores cualificados, completaban una serie de cuestionarios y valoraciones.

El proyecto DADOS se ha desarrollado cumpliendo las recomendaciones éticas de la Declaración de Helsinki 1961 (última revisión de Fortaleza, Brasil, 2013) y su protocolo de investigación ha sido aprobado por la Comisión Deontológica de la Universitat Jaume I de Castellón. Todos los participantes y sus padres o tutores legales recibieron una explicación detallada del protocolo de investigación y firmaron un consentimiento informado.

\section{Instrumentos}

- Estrés social

El estrés social se evaluó utilizando el nivel 3 de la versión española del cuestionario BASC (Behavior Assessment System for Children), diseñado para adolescentes de 14 años (Reynolds y Kamphaus 2004). El cuestionario presenta 185 ítems organizados en escalas clínicas y adaptativas. Los 
estudiantes fueron dicotomizados según su nivel en la escala clínica de estrés social basada en puntuaciones $T$ (normal $<60$ frente a elevado $\geq 60$ ).

\section{- Rendimiento académico}

El rendimiento académico se estableció a partir de las calificaciones finales obtenidas por cada uno de los estudiantes en el curso anterior $\left(1 .^{\circ}\right.$ de ESO). Cada adolescente aportó el certificado original de notas proporcionado por su centro educativo. Para este análisis exclusivamente se utilizaron las calificaciones finales correspondientes a las asignaturas: castellano, valenciano, inglés, matemáticas y educación física, así como la calificación media global calculada como la media aritmética de todas las asignaturas (geografía e historia, ciencias naturales, matemáticas, castellano, valenciano, inglés y educación física).

\section{Covariables}

El sexo fue reportado por los participantes en el estudio. Asimismo, todos reportaron su nivel de desarrollo madurativo a partir de la observación de gráficos estándar sobre las características sexuales primarias y secundarias según el protocolo establecido por Tanner y Whitehouse (1976). Para ello se evaluó en una escala del uno al cinco el nivel de desarrollo del vello púbico y de las mamas. El valor más alto de estos dos componentes fue utilizado para establecer el nivel de desarrollo madurativo general.

\section{Análisis estadístico}

Las características de la muestra de estudio se presentan como media y desviación estándar o frecuencia y porcentaje para variables continuas y categóricas, respectivamente. La normalidad de los datos se comprobó de forma gráfica (histogramas), así como mediante el test de Kolmogorov-Smirnov. Todas las variables mostraron una distribución normal. Los análisis preliminares mostraron que no existía interacción del sexo con el estrés social en relación con el rendimiento académico (todas $p>0,05$ ), por lo que los análisis se realizaron con la muestra completa.

Las diferencias entre sexos se evaluaron empleando la prueba de T-Student para muestras independientes para variables continuas y la prueba chi-cuadrado para las variables categóricas.

Para examinar la relación entre el estrés social y el rendimiento académico se realizaron correlaciones parciales ajustando por sexo y desarrollo madurativo. Asimismo, se realizaron análisis de regresión lineal para examinar la asociación entre el estrés social y el rendimiento académico ajustando por sexo y nivel de desarrollo madurativo.

La muestra se dicotomizó en base al nivel de estrés social de los estudiantes (normal<60 frente a elevado $\geq 60$ ) y se analizaron las diferencias en el rendimiento académico empleando análisis de covarianza (ANCOVA) ajustados por sexo y nivel de desarrollo madurativo. Todos los análisis fueron realizados con el programa estadístico IBM SPSS 22.0 (Armonk, NY: IBM Corp.) y se estableció un nivel de significación de $p<0,05$. 


\section{Resultados}

Las características de la muestra se presentan en la tabla 1. Las chicas mostraron un mayor nivel de estrés social que los chicos (media aritmética: 47,8 frente a 44,6; $p=0,003$ ). El $3 \%$ de los chicos y el $11 \%$ de las chicas presentaron niveles de estrés social elevado. Respecto al rendimiento académico, no se encontraron diferencias estadísticamente significativas entre chicos y chicas (todas $p>0,05$ ).

Tabla 1

Características de la muestra de estudio

\begin{tabular}{|c|c|c|c|c|}
\hline & Todos & Chicos & Chicas & p \\
\hline $\mathrm{n}$ & 269 & 140 & 129 & \\
\hline \multicolumn{5}{|l|}{ Demográficos } \\
\hline Edad (años) & $13,9 \pm 0,3$ & $13,9 \pm 0,3$ & $13,9 \pm 0,3$ & 0,903 \\
\hline $\begin{array}{l}\text { Desarrollo madurativo } \\
(\mathrm{II}-\mathrm{V})(\%)\end{array}$ & $\begin{array}{c}21(8) \\
92(34) \\
130(48) \\
26(10) \\
\end{array}$ & $\begin{array}{l}14(10) \\
45(32) \\
61(44) \\
20(14) \\
\end{array}$ & $\begin{array}{c}7(5) \\
47(36) \\
69(54) \\
6(5)\end{array}$ & $0,019^{*}$ \\
\hline Estrés social $^{a}$ & $46,1 \pm 8,7$ & $44,6 \pm 6,5$ & $47,8 \pm 10,3$ & $0,003^{* *}$ \\
\hline Elevado $(\geq 60)$ & $18(7)$ & $4(3)$ & $14(11)$ & $0,009^{* *}$ \\
\hline \multicolumn{5}{|l|}{ Rendimiento académico $^{\mathrm{b}}$} \\
\hline Matemáticas & $6,8 \pm 1,6$ & $7,0 \pm 1,6$ & $6,7 \pm 1,6$ & 0,196 \\
\hline Castellano & $6,9 \pm 1,5$ & $6,7 \pm 1,5$ & $7,1 \pm 1,5$ & 0,075 \\
\hline Valenciano & $6,8 \pm 1,5$ & $6,6 \pm 1,5$ & $6,9 \pm 1,5$ & 0,168 \\
\hline Inglés & $7,2 \pm 1,7$ & $7,1 \pm 1,7$ & $7,4 \pm 1,8$ & 0,248 \\
\hline Educación física & $8,1 \pm 1,1$ & $8,0 \pm 1,1$ & $8,1 \pm 1,1$ & 0,516 \\
\hline Calificación media & $7,1 \pm 1,3$ & $7,1 \pm 1,3$ & $7,2 \pm 1,3$ & 0,420 \\
\hline
\end{tabular}

Datos diferencias entre na media de 50 y estándar de 10 puntos.

${ }^{\mathrm{b}}$ Resultados del rendimiento académico basados en una escala de 0 a 10.

La tabla 2 muestra las correlaciones parciales entre el estrés social y el rendimiento académico en adolescentes, ajustando por sexo y nivel de desarrollo madurativo. El nivel de estrés social estuvo inversamente relacionado con todos los indicadores de rendimiento académico ( $r$ desde $-0,242$ a $-0,121$; todos $p<0,05$ ).

Tabla 2

Correlaciones parciales entre las variables de estudio, ajustando por se xo y nivel de desarrollo madurativo $(n=269)$

\begin{tabular}{|c|c|c|c|c|c|c|c|}
\hline & & Matemáticas & Castellano & Valenciano & Inglés & $\begin{array}{l}\text { Educación } \\
\text { física }\end{array}$ & $\begin{array}{l}\text { Calificación } \\
\text { media }\end{array}$ \\
\hline $\begin{array}{l}\text { Estrés } \\
\text { social }\end{array}$ & $\begin{array}{l}r \\
p\end{array}$ & $\begin{array}{l}-0,184 \\
\mathbf{0 , 0 0 3}\end{array}$ & $\begin{array}{l}-0,121 \\
\mathbf{0 , 0 4 8}\end{array}$ & $\begin{array}{l}-0,191 \\
\mathbf{0 , 0 0 2}\end{array}$ & $\begin{array}{l}-0,242 \\
<0,001\end{array}$ & $\begin{array}{l}-0,132 \\
\mathbf{0 , 0 3 1}\end{array}$ & $\begin{array}{l}-0,200 \\
<0,001\end{array}$ \\
\hline
\end{tabular}

r: coeficiente de correlación de Pearson. Los resultados significativos se muestran en negrita $(p<0,05)$. 
La tabla 3 presenta los análisis de regresión lineal entre el estrés social y el rendimiento académico en adolescentes, ajustando por sexo y desarrollo madurativo. El nivel de estrés social estuvo inversamente asociado con todos los indicadores de rendimiento académico ( $\beta$ desde $-0,246$ a $-0,123$; todos $p<0,05)$.

Tabla 3

Regresión lineal examinando la asociación entre el estrés social y el rendimiento académico en adolescentes, ajustando por sexo y nivel de desarrollo madurativo

\begin{tabular}{lcc}
\hline & $\beta$ & $p$ \\
\hline Matemáticas & $-0,187$ & $\mathbf{0 , 0 0 3}$ \\
\hline Castellano & $-0,123$ & $\mathbf{0 , 0 4 8}$ \\
\hline Valenciano & $-0,195$ & $\mathbf{0 , 0 0 2}$ \\
\hline Inglés & $-0,246$ & $\mathbf{0 , 0 0 0}$ \\
\hline Educación física & $-0,135$ & $\mathbf{0 , 0 3 1}$ \\
\hline Calificación media & $-0,203$ & $\mathbf{0 , 0 0 1}$ \\
\hline
\end{tabular}

ß: coeficiente estandarizado de regresión lineal. Los resultados significativos se muestran en negrita.

Los análisis de covarianza, examinando las diferencias en el rendimiento académico en base al nivel de estrés social de los adolescentes, ajustando por sexo y nivel de desarrollo madurativo, se muestran en la tabla 4 . Los estudiantes con un estrés social elevado $(\geq 60)$ presentaron un menor rendimiento académico (todos $p<0,05$ ), excepto en castellano $(p=0,153)$.

Tabla 4

Análisis de covarianza examinado diferencias en el rendimiento académico por categorías de estrés social, ajustando por sexo y nivel de desarrollo madurativo

\begin{tabular}{lccc} 
& \multicolumn{2}{c}{ Estrés social } \\
\cline { 2 - 4 } & Normal $(<60)$ & Elevado $(\geq 60)$ & $\mathrm{p}$ \\
\hline $\mathrm{n}$ & 251 & 18 & $\mathbf{0 , 0 1 6}$ \\
\hline Matemáticas & $6,9 \pm 1,6$ & $5,9 \pm 1,3$ & 0,153 \\
\hline Castellano & $6,9 \pm 1,5$ & $6,5 \pm 1,5$ & $\mathbf{0 , 0 2 0}$ \\
\hline Valenciano & $6,8 \pm 1,5$ & $6,1 \pm 1,4$ & $\mathbf{0 , 0 0 1}$ \\
\hline Inglés & $7,3 \pm 1,7$ & $6,0 \pm 1,8$ & $\mathbf{0 , 0 2 9}$ \\
\hline Educación física & $8,1 \pm 1,1$ & $7,6 \pm 1,3$ & $\mathbf{0 , 0 0 6}$ \\
\hline Calificación media & $7,2 \pm 1,3$ & $6,4 \pm 1,1$ & \\
\hline
\end{tabular}

Datos presentados como media \pm desviación estándar. Los resultados significativos se muestran en negrita $(p<0,05)$. 


\section{Discusión y conclusiones}

Los principales hallazgos de nuestro estudio ponen de manifiesto una relación inversa entre el nivel de estrés social y el rendimiento académico en estudiantes de $2 .^{\circ}$ de ESO. Del mismo modo, nuestros resultados muestran que los estudiantes con un mayor nivel de estrés presentaban un menor rendimiento académico. Estos resultados complementan la evidencia científica previa sobre la relación entre el estrés y el rendimiento académico en adolescentes.

En base a nuestro conocimiento, no existen estudios previos que hayan analizado la asociación entre el estrés de tipo social y el rendimiento académico en adolescentes, lo que hace imposible la comparación con estudios similares. Sin embargo, existe literatura científica que examina la asociación entre otros tipos de estrés (por ejemplo, a cadémico) y el rendimiento académico en este grupo de población, así como en otros grupos de edad. Nuestros resultados coinciden con los obtenidos por Lee (2017), quien indicó que el estrés se asociaba positivamente con el fracaso académico percibido e inversamente con el rendimiento académico en jóvenes. De igual modo, Grasses y Rigo (2010) examinaron la influencia del estrés en el rendimiento académico en 471 adolescentes y mostraron que los estudiantes con niveles elevados de estrés presentaban un menor rendimiento académico. Asimismo, Fernández de Castro de León y Luévano Flores (2018) Ilevaron a cabo un estudio en 179 jóvenes que indicaba una asociación negativa entre el grado de estrés académico y el rendimiento académico. Por último, en un estudio de revisión reciente, Ye, Posada y Liu (2019) revelaron una relación inversa entre el estrés y el rendimiento académico en adolescentes chinos. Sin embargo, en un estudio llevado a cabo en una muestra muy pequeña compuesta por 20 jóvenes, Domínguez Castillo, Guerrero Walker y Domínguez Castillo (2015) analizaron la relación entre estrés académico y rendimiento académico y mostraron una falta de asociación.

Si bien la relación negativa encontrada en estudios previos entre el estrés académico y el rendimiento académico podría explicarse parcialmente por las altas cargas de tareas evaluables que deben abordar los estudiantes, así como por las posibles exigencias del profesorado (Pulido et al. 2011), especulamos que la relación entre el estrés social y el rendimiento académico encontrada en el presente estudio podría deberse a la presión social a la que se encuentran sometidos estos adolescentes. De hecho, esta presión podría influir sobre el autoconcepto físico y su imagen corporal (McCabe y Ricciardelli 2004; Gervilla 2002), lo que a su vez podría afectar a su rendimiento académico (Larsen, Kleinjan, Engels, Fisher y Hermans 2014; Griffiths, Parsons y Hill 2010). La evidencia científica previa sugiere que la relación entre el estrés y la cognición depende de la magnitud e intensidad del estrés, así como de su origen y duración, revelando una influencia negativa del estrés sobre la función cognitiva (Lupien, McEwen, Gunnar y Heim 2009; Sandi 2013), que podría derivar en consecuencias negativas para el rendimiento académico de los adolescentes. Además, estar bajo niveles de estrés elevados podría reducir la motivación, la concentración y la atención, incrementar el absentismo escolar, los síntomas depresivos y la ansiedad, así como producir alteraciones del sueño y de la alimentación (Maturana y Vargas 2015), lo que podría afectar al rendimiento académico (Adelantado Renau et al. 2019; Adelantado Renau et al. 2018).

Nuestros hallazgos podrían tener implicaciones significativas desde el punto de vista educativo y de salud pública dada la alta prevalencia de estrés en adolescentes (Gaeta González y Martín Hernández 2009). Debido a la gran influencia del rendimiento académico en la empleabilidad (French, Homer, Popovici y Robins 2015) y el estado de salud en la etapa adulta (Lê-Scherban, Diez Roux, Li y Morgenstern 2014), los programas escolares y de salud pública deberían trabajar elementos 
sociales y contemplar actividades que fomenten la relajación y la práctica de actividad física con el objetivo de mejorar el bienestar psicológico de los adolescentes (Morilla Cabezas 2001).

\section{Limitaciones y fortalezas}

Nuestros resultados han de ser interpretados con cautela debido a que se trata de un estudio de diseño transversal y no es posible establecer relaciones causa-efecto. Sin embargo, nuestro estudio presenta diversas fortalezas entre las que cabe destacar la homogeneidad de los participantes en relación a su edad, el uso de un cuestionario validado para evaluar el estrés social (BASC) y la inclusión en los análisis de variables de gran relevancia tanto para el estrés social como para el rendimiento académico, así como el ajuste de los análisis por nivel de desarrollo madurativo.

\section{Conclusiones}

En conclusión, el nivel de estrés social parece estar inversamente asociado con el rendimiento académico en estudiantes de $2 .^{\circ}$ de ESO. Los programas escolares enfocados a la mejora del rendimiento académico deberían tener en cuenta los posibles efectos negativos derivados de los altos niveles de estrés social en la adolescencia. Se requieren futuros estudios que clarifiquen la relación entre el estrés social y el rendimiento académico en la etapa escolar.

\section{Agradecimientos}

El proyecto DADOS está financiado por el Ministerio de Economía y Competitividad (DEP2013-45515-R) y por la Universitat Jaume I de Castellón (P1-1A2015-05). Este trabajo ha sido parcialmente financiado por una beca de investigación Sunny Sport de la compañía Schweppes Suntory Spain. M. A. R. cuenta con una ayuda predoctoral para la formación de personal investigador de la Universitat Jaume I (PREDOC/2015/13).

\section{Referencias bibliográficas}

Adelantado Renau, Mireia, María Reyes Beltrán Valls, Irene Esteban Cornejo, Vicente Martínez Vizcaíno, Alba María Santaliestra Pasías y Diego Moliner Urdiales. 2018. "The influence of adherence to the Mediterranean diet on academic performance is mediated by sleep quality in adolescents». Acta Paediatrica, International Journal of Paediatrics, 108(2): 339-346.

Adelantado Renau, Mireia, María Reyes Beltrán Valls, Jairo Hidalgo Migueles, Enrique García Artero, Alejandro Legaz Arrese, Ana Capdevila Seder y Diego Moliner Urdiales. 2019. "Associations between objectively measured and self-reported sleep with academic and cognitive performance in adolescents: DADOS study». Journal of Sleep Research, 28(4): e12811.

Cruz Fabio, Josiane O. Duarte, Rodrigo M. Leão, Luiz F. V. Hummel, Cleopatra S. Planeta y Carlos César Crestani. 2015. «Adolescent vulnerability to cardiovascular consequences of chronic social stress: Immediate and long-term effects of social isolation during adolescence». Developmental Neurobiology, 76(1): 34-46. 
Domínguez Castillo, Rodrigo Arturo, Julieta Gladys Guerrero Walker y José Gabriel Domínguez Castillo. 2015. «Influencia del estrés en el rendimiento académico de un grupo de estudiantes universitarios». Educación y ciencia, 4(43): 31-40.

Fernández de Castro de León, Javier y Edith Luévano Flores. 2018. «Influencia del estrés académico sobre el rendimiento escolar en educación media superior». Revista Panamericana de Pedagogía, 26: 97-117.

French, Michael T., Jenny F. Homer, loana Popovici y Philip K. Robins. 2015. «What you do in high school matters: high school GPA, educational attainment, and labor market earnings as a young adult». Eastern Economic Journal, 41(3): 370-386.

Gaeta González, Martha Leticia y Pilar Martín Hernández. 2009. «Estrés y adolescencia: estrategias de afrontamiento y autorregulación en el contexto escolar». STVDIVM: Revista de Humanidades, 15: 327-344.

Gervilla Castillo, Enrique. 2002. "La tiranía de la belleza, un problema educativo hoy. La estética del cuerpo como valor y problema». Teoría de la Educación, 14: 185-206.

Grases Colom, Glòria y Eduard Rigo Carratalà. 2010. «Estudio de la influencia de la ansiedad y el estrés en el rendimiento académico vs. la influencia del rendimiento académico en los niveles de ansiedad y estrés». Educació $i$ Cultura, 21: 97-116.

Griffiths, Lucy J., Tessa J. Parsons y Andrew J. Hill. 2010. «Self-esteem and quality of life in obese children and adolescents: A systematic review». International Journal of Pediatric Obesity, 5(4): 282-304.

Larsen, Junilla K., Marloes Kleinjan, Rutger C. M. E. Engels, Jennifer O. Fisher y Roel C. J. Hermans. 2014. "Higher Weight, Lower Education: A Longitudinal Association Between Adolescents' Body Mass Index and Their Subsequent Educational Achievement Level?». Journal of School Health, 84(12): 769-776.

Lee, Wincy Wing Szel. 2017. «Relationships among grit, academic performance, perceived academic failure, and stress in associate degree students». Journal of Adolescence, 60: 148-152.

Lê-Scherban, Félice, Ana V. Diez Roux, Yun Li y Harold Morgenstern. 2014. «Does academic achievement during childhood and adolescence benefit later health?». Annals of Epidemiology, 24(5): 344-355.

Lupien, Sonia J., Bruce S. McEwen, Megan R. Gunnar y Christine Heim. 2009. «Effects of stress throughout the lifespan on the brain, behaviour and cognition». Nature Reviews Neuroscience, 10(6): 434-445.

Maturana Hurtado, Alejandro y S. Ana Vargas. 2015. «El estrés escolar». Revista Médica Clínica Las Condes, 26(1): 34-41.

McCabe, Marita y Lina Ricciardelli. 2004. "Body image dissatisfaction among males across the life spam. A review of past literature». Journal of Psychosomatic Research, 56: 675-685.

Morilla Cabezas, Miguel. 2001. «Beneficios psicológicos de la actividad física y el deporte». Efdeportes, 43(1).

Ortega, Francisco, Jonatan Ruiz, Manuel Castillo y Michael Sjöström. 2008. «Physical fitness in childhood and adolescence: a powerful marker of health». International Journal of Obesity, 32(1): 1-11.

Pulido Rull, Marco Antonio, María Luisa Serrano Sanchez, Estefanía Valdés Cano, María Teresa Chávez Méndez, Pamela Hidalgo Montiel y Fernando Vera García. 2011. «Estrés académico en estudiantes universitarios». Revista Psicología y Salud, 21(1): 31-37.

Reynolds, Cecil R. y Randy W. Kamphaus. 2004. Behavior assessment system for children. Circle Pines, MN: American Guidance Service, Inc.

Sandi, Carmen. 2013. «Stress and cognition». Wiley Interdisciplinary Reviews: Cognitive Science, 4: 245-261. 
Tanner, James M. y Reg H. Whitehouse. 1976. «Clinical longitudinal standards for height, weight, height velocity, weight velocity, and stages of puberty». Archives of Disease in Childhood, 51(3): 170-179.

Vales, Lisandro. 2009. Resiliencia y Vulnerabilidad en un Proyecto Social. El funcionamiento mental impulsivo y el apoyo social en la red vincular en niños de 10 a 14 años en situaciones de vulnerabilidad social estresantes. Buenos Aires, Argentina: Universidad de Favaloro.

Van Roekel, Eeske, Thao Ha, Maaike Verhagen, Emmanuel Kuntsche, Ron Scholte y Rutger Engels. 2015. "Social stress in early adolescents' daily lives: Associations with affect and loneliness». Journal of Adolescence, 45: 274-283.

Ye, Lin, Alexandria Posada y Yangyang Liu. 2019. "A review on the relationship between Chinese adolescents' stress and academic achievement». New Directions for Child and Adolescent Development, 163: 81-95.

Yeresyan, Iren y Arnold Lohaus. 2014. "Stress and wellbeing among Turkish and German adolescents living in rural and urban areas». Rural and Remote Health, 14: 2695. 